\title{
The Emotional Impact of Mental Illness on Mental Health Provider
}

\author{
Raafat Aburumman*1, Maher Al-Asasleh ${ }^{1}$, Amjad Jumaiann ${ }^{1}$ and Jed Megan ${ }^{2}$ \\ ${ }^{1}$ Royal Medical Services, Jordan \\ ${ }^{2}$ Department of psychiatry, Michigan State University, USA
}

Received: 監: September 06, 2018; Published: 㘹 September 21, 2018

*Corresponding author: Raafat Aburumman, Royal Medical Services, Jordan

\begin{abstract}
Mental health providers are likely to develop negative emotions towards patients with mental diseases. The objectives of this study were to explore the bothering levels of mental patients, and what emotion feelings that were developed by American and Arabian mental health providers towards the patients they are treating. To achieve these objectives, a questionnaire was developed that consisted of demographic part, scoring of bothering levels, emotional feelings, and mechanisms of interactions with patients in case of the existence of such emotions. The questionnaire was uploaded to Survey Monkey for data collection. The data was presented as frequencies and percentages. Study findings showed the existence of bothering levels according to mental diseases. In the majority of cases, both Americans and Arabians exhibited empathy towards patients with mental diseases. In case of having such an emotional feeling that may interfere with treatment plans, various mechanisms were involved such as reviewing literature or consulting other colleagues. Taken together, both Americans and Arabians developed bothering levels of actions of mental patients and exhibited different levels of empathy towards those patients.
\end{abstract}

Keywords: Mental Health Providers; Patients with Mental Disease; Empathy; Emotional Feelings; Americans; Arabians

\section{Introduction}

The present study seeks the emotional impacts of mental illness on mental health providers among American and Arabian mental health providers. Across the literature, mental health providers were found to express negative emotions towards patients with mental diseases. In this context, Lauber et al. [1] conducted a study to explore the opinions of health professional about patients with diseases. A five point Likert scale was used to satisfy this purpose. The data of that study showed that mental health professionals described those patients with negative traits such as unpredictable, bedraggled, weird, threatening, and dangerous. It was also shown that mental health providers have negative attitudes towards patients with mental diseases. In another study, Deans and Meocivic [2] explored the perceptions of psychiatric nurses in Australia towards patients with borderline personality disorder (BPD). The results indicated that psychiatric nurses developed negative emotions towards such patients as being manipulative (88\%) and engaging in emotional blackmail (51\%), and nuisances (38\%). About one third of participants frankly reported that they felt angry by BPD patients. These findings have been further confirmed by the study of James and Cowman [3] who found that psychiatric nurses in Ireland in general considered patients with BPD are not easy to look after.
Servais and Saunders [4] conducted a study to explore the opinions of clinical psychologists in the United States about patients with mental diseases such as depression, BPD, and schizophrenia. The results showed that mental patients were perceived as less than other persons in public and rated as ineffective and undesirable. The study of Ucok et al. [5] showed that psychiatrists exhibited negative appraisals about the ability of schizophrenic patients to participate in a collaborative therapeutic relationship. The findings of that study revealed that more than $80 \%$ of psychiatrists do not routinely inform their schizophrenic patients about their disease due to the consideration that those patients do not understand their diagnosis. Psychiatric nurses in Sweden exhibited similar trends towards schizophrenic patients as unpredictable, hard to talk to, unusual, and unlikely to recover [6].

Empathy is one of complicated emotions that involve the feeling of others in an attempt of sharing and understanding their emotions and the readiness to help them [7-9]. Furthermore, empathy is considered as a complex emotion involving sociocognitive competence with the involvement of different components that interact including affective sharing and perspective taking [10-12]. 


\section{Methods and Subjects}

\section{Study Design}

A comparative study design for the emotional impact of mental Illness on mental health providers of Americans and Arabians was conducted.

\section{Study Participants}

The study included mental health providers from American origins (46), and Arabian origins (88).

\section{Study Instruments}

The study instrument has been developed through reviewing several studies and its paragraphs have been extracted and formulated to include the following parts: demographic part which included age, gender, education and ethnical background; scoring system to express the bothering level of mental health providers towards patients with mental diseases. This system ranged from 1-10, 1 indicated the least bothering level, whereas 10 indicates the maximal bothering level; emotional feelings of mental health providers towards patients with mental diseases. A total of 8 emotional feelings were investigated that include: anger, frustration, sadness, guilty, empathy, disgust, fear or nervousness, no particular feelings. The last part of the instrument was made to investigate the mechanisms of reactions of mental health providers in case their emotional feelings may interfere with their treatment to their patients.

\section{Study Procedure}

The present study was approved by IRB (Institutional Review Board at Royal Medical Services). The study instrument as previously described was constructed and uploaded to Survey Monkey to collect data from participants. After data had been collected and analyzed by Survey Monkey, the article was prepared.

\section{Results}

The data presented in Table (1) showed that the distribution of age in American participants peaked in the age intervals 30-39 years $(28.26 \%)$, and $50-59$ years $(26.09 \%)$. On the other hand, the majority of Arabian participants peaked in the age intervals $20-29$ years (44.83\%), and 30-39 years (36.78\%). Regarding the gender, in both types of participants, females were more frequent than males. All participants were Americans and Arabians, no other ethnic backgrounds were included in this study. Regarding educational level, the American participants had $\mathrm{PhD}$ (34.78\%) and medical doctors (56.52\%). On the other hand, 37.5\% of Arabian participants had medical doctor qualifications, and $22.37 \%$ had bachelor's degree.

Table 1: Frequency and distribution of demographic variables by study participants.

\begin{tabular}{|c|c|c|c|c|}
\hline \multirow{2}{*}{ Variable } & \multicolumn{2}{|c|}{ American } & \multicolumn{2}{|c|}{ Arabian } \\
\hline & $\mathbf{N}$ & Percentage (\%) & $\mathbf{N}$ & Percentage (\%) \\
\hline \multicolumn{5}{|c|}{ Age (years) } \\
\hline $20-29$ & 5 & $10.87 \%$ & 39 & $44.83 \%$ \\
\hline $30-39$ & 13 & $28.26 \%$ & 32 & $36.78 \%$ \\
\hline $40-49$ & 8 & $17.39 \%$ & 8 & $9.20 \%$ \\
\hline $50-59$ & 12 & $26.09 \%$ & 5 & $5.75 \%$ \\
\hline $60-69$ & 8 & $17.39 \%$ & 3 & $3.45 \%$ \\
\hline$>70$ & 0 & $0.0 \%$ & 0 & $0 \%$ \\
\hline Total & 46 & $34.59 \%$ & 87 & $65.41 \%$ \\
\hline \multicolumn{5}{|c|}{ Gender } \\
\hline Male & 22 & $47.83 \%$ & 41 & $46.59 \%$ \\
\hline Female & 24 & $52.17 \%$ & 47 & $53.41 \%$ \\
\hline Total & 46 & $34.33 \%$ & 88 & $65.67 \%$ \\
\hline \multicolumn{5}{|c|}{ Ethnic background } \\
\hline American & 46 & $100 \%$ & 0 & $0 \%$ \\
\hline African & 0 & $0 \%$ & 0 & $0 \%$ \\
\hline Asian & 0 & $0 \%$ & 0 & $0 \%$ \\
\hline Latino & 0 & $0 \%$ & 0 & $0 \%$ \\
\hline European & 0 & $0 \%$ & 0 & $0 \%$ \\
\hline Arabian & 0 & $0 \%$ & 88 & $100 \%$ \\
\hline Total & 46 & $34.33 \%$ & 88 & $65.67 \%$ \\
\hline \multicolumn{5}{|c|}{ Education } \\
\hline MA/MS & 4 & $8.7 \%$ & 17 & $19.32 \%$ \\
\hline Ph.D/ED.D & 16 & $34.78 \%$ & 18 & $20.45 \%$ \\
\hline MD/DO & 26 & $56.52 \%$ & 33 & $37.50 \%$ \\
\hline BS & 0 & $0 \%$ & 20 & $22.37 \%$ \\
\hline Total & 46 & $34.33 \%$ & 88 & $65.67 \%$ \\
\hline
\end{tabular}

Cite this article: Raafat A, Maher Al-A, Amjad J, Jed M. The Emotional Impact of Mental Illness on Mental Health Provider. Biomed J Sci\&Tech 
Table 2: Scoring of the reactions of mental health providers towards psychological cases of patients.

\begin{tabular}{|c|c|c|c|c|c|c|c|c|c|c|c|c|c|c|c|c|}
\hline \multirow[t]{3}{*}{ Degree } & \multicolumn{4}{|c|}{ Suicide attempts } & \multicolumn{4}{|c|}{ Depressive disorders } & \multicolumn{4}{|c|}{ Psychosis } & \multicolumn{4}{|c|}{ Sexual abuse } \\
\hline & \multicolumn{2}{|c|}{ Americans } & \multicolumn{2}{|c|}{ Arabian } & \multicolumn{2}{|c|}{ Americans } & \multicolumn{2}{|c|}{ Arabian } & \multicolumn{2}{|c|}{ Americans } & \multicolumn{2}{|c|}{ Arabian } & \multicolumn{2}{|c|}{ Americans } & \multicolumn{2}{|c|}{ Arabian } \\
\hline & $\mathrm{N}$ & $\%$ & $\mathrm{~N}$ & $\%$ & $\mathrm{~N}$ & $\%$ & $\mathrm{~N}$ & $\%$ & $\mathrm{~N}$ & $\%$ & $\mathrm{~N}$ & $\%$ & $\mathrm{~N}$ & $\%$ & $\mathrm{~N}$ & $\%$ \\
\hline 1 & 0 & $0 \%$ & 7 & $9.59 \%$ & 1 & $2.17 \%$ & 0 & $0 \%$ & 1 & $2.17 \%$ & 6 & $8.45 \%$ & 0 & $0 \%$ & 7 & $9.59 \%$ \\
\hline 2 & 1 & $2.17 \%$ & 6 & $8.22 \%$ & 7 & $15.22 \%$ & 7 & $9.86 \%$ & 3 & $6.52 \%$ & 5 & $7.04 \%$ & 1 & $2.17 \%$ & 4 & 5.48 \\
\hline 3 & 2 & $4.35 \%$ & 7 & $9.59 \%$ & 3 & $6.25 \%$ & 7 & $9.86 \%$ & 4 & $8.70 \%$ & 9 & $12.08 \%$ & 3 & $6.52 \%$ & 4 & 5.48 \\
\hline 4 & 2 & $4.35 \%$ & 6 & $8.22 \%$ & 2 & $4.35 \%$ & 6 & $8.45 \%$ & 9 & $19.57 \%$ & 5 & $7.04 \%$ & 8 & $17.39 \%$ & 6 & $8.22 \%$ \\
\hline 5 & 3 & $6.52 \%$ & 2 & $2.74 \%$ & 5 & $10.87 \%$ & 11 & $15.49 \%$ & 9 & $19.57 \%$ & 6 & $8.45 \%$ & 11 & $23.91 \%$ & 5 & $6.85 \%$ \\
\hline 6 & 2 & $4.35 \%$ & 5 & $6.85 \%$ & 11 & $23.91 \%$ & 6 & $8.45 \%$ & 3 & $6.52 \%$ & 10 & $14.08 \%$ & 4 & $8.70 \%$ & 2 & $2.74 \%$ \\
\hline 7 & 3 & $6.52 \%$ & 5 & $6.85 \%$ & 5 & $10.87 \%$ & 10 & $14.08 \%$ & 6 & $13.04 \%$ & 14 & $19.72 \%$ & 4 & $8.70 \%$ & 5 & $6.85 \%$ \\
\hline 8 & 6 & $13.04 \%$ & 4 & $5.48 \%$ & 10 & $21.74 \%$ & 13 & $18.31 \%$ & 9 & $19.57 \%$ & 9 & $12.08 \%$ & 13 & $28.26 \%$ & 23 & $31.51 \%$ \\
\hline 9 & 23 & $50 \%$ & 14 & $19.18 \%$ & 2 & $4.35 \%$ & 10 & $14.08 \%$ & 2 & $4.35 \%$ & 6 & $8.45 \%$ & 2 & $4.35 \%$ & 9 & $12.33 \%$ \\
\hline 10 & 4 & $8.70 \%$ & 17 & $23.29 \%$ & 0 & $0 \%$ & 1 & $1.41 \%$ & 0 & $0 \%$ & 1 & $1.41 \%$ & 0 & $0 \%$ & 8 & $10.96 \%$ \\
\hline
\end{tabular}

\begin{tabular}{|c|c|c|c|c|c|c|c|c|c|c|c|c|c|c|c|}
\hline \multicolumn{4}{|c|}{ Physical abuse } & \multicolumn{4}{|c|}{ Substance Abuse } & \multicolumn{4}{|c|}{ Bipolar Disorder } & \multicolumn{4}{|c|}{ Anxiety Disorders } \\
\hline \multicolumn{2}{|c|}{ American } & \multicolumn{2}{|c|}{ Arabian } & \multicolumn{2}{|c|}{ American } & \multicolumn{2}{|c|}{ Arabian } & \multicolumn{2}{|c|}{ American } & \multicolumn{2}{|c|}{ Arabian } & \multicolumn{2}{|c|}{ American } & \multicolumn{2}{|c|}{ Arabian } \\
\hline $\mathrm{N}$ & $\%$ & $\mathrm{~N}$ & $\%$ & $\mathrm{~N}$ & $\%$ & $\mathrm{~N}$ & $\%$ & $\mathrm{~N}$ & $\%$ & $\mathrm{~N}$ & $\%$ & $\mathrm{~N}$ & $\%$ & $\mathrm{~N}$ & $\%$ \\
\hline 0 & $0 \%$ & 4 & $5.63 \%$ & 0 & $0 \%$ & 4 & $5.56 \%$ & 1 & $2.17 \%$ & 3 & $4.17 \%$ & 1 & $2.17 \%$ & 2 & $2.78 \%$ \\
\hline 2 & $4.35 \%$ & 7 & $9.86 \%$ & 3 & $6.52 \%$ & 6 & $8.33 \%$ & 0 & $0 \%$ & 10 & $13.89 \%$ & 9 & $19.57 \%$ & 13 & $18.06 \%$ \\
\hline 3 & $6.52 \%$ & 7 & 9.86 & 11 & $23.91 \%$ & 8 & $11.11 \%$ & 8 & $17.39 \%$ & 10 & $13.89 \%$ & 10 & $21.47 \%$ & 5 & $6.94 \%$ \\
\hline 8 & $17.39 \%$ & 3 & $4.23 \%$ & 6 & $13.04 \%$ & 8 & $11.11 \%$ & 9 & $19.57 \%$ & 6 & $8.33 \%$ & 6 & $13.04 \%$ & 13 & $18.06 \%$ \\
\hline 11 & $23.91 \%$ & 7 & $9.86 \%$ & 4 & $8.70 \%$ & 15 & $20.83 \%$ & 10 & $21.74 \%$ & 19 & $26.39 \%$ & 4 & $8.70 \%$ & 13 & $18.06 \%$ \\
\hline 4 & $8.70 \%$ & 8 & $11.27 \%$ & 6 & $13.04 \%$ & 9 & $12.50 \%$ & 3 & $6.52 \%$ & 14 & $19.44 \%$ & 7 & $15.22 \%$ & 4 & $5.56 \%$ \\
\hline 9 & 18.575 & 8 & $11.27 \%$ & 11 & $23.91 \%$ & 5 & $6.94 \%$ & 12 & $26.09 \%$ & 3 & $4.17 \%$ & 9 & $19.57 \%$ & 14 & $19.44 \%$ \\
\hline 8 & $17.39 \%$ & 19 & $26.76 \%$ & 2 & $4.35 \%$ & 8 & $11.11 \%$ & 2 & $4.35 \%$ & 0 & $0 \%$ & 0 & $0 \%$ & 3 & $4.17 \%$ \\
\hline 1 & 2.17 & 4 & $5.63 \%$ & 2 & $4.35 \%$ & 5 & $6.94 \%$ & 1 & $2.17 \%$ & 5 & $6.94 \%$ & 0 & $0 \%$ & 4 & $5.56 \%$ \\
\hline 0 & $0 \%$ & 4 & $5.63 \%$ & 1 & $2.17 \%$ & 4 & $5.56 \%$ & 0 & $0 \%$ & 2 & $2.78 \%$ & 0 & $0 \%$ & 1 & $1.39 \%$ \\
\hline
\end{tabular}

\begin{tabular}{|c|c|c|c|c|c|c|c|c|c|c|c|c|c|c|c|}
\hline \multicolumn{4}{|c|}{ Post- Traumatic Stress Disorder } & \multicolumn{4}{|c|}{ Childhood Psychiatric Disorders } & \multicolumn{4}{|c|}{ Personality Disorders } & \multicolumn{4}{|c|}{ Deliberate Self-Harm } \\
\hline \multicolumn{2}{|c|}{ American } & \multicolumn{2}{|c|}{ Arabian } & \multicolumn{2}{|c|}{ American } & \multicolumn{2}{|c|}{ Arabian } & \multicolumn{2}{|c|}{ American } & \multicolumn{2}{|c|}{ Arabian } & \multicolumn{2}{|c|}{ American } & \multicolumn{2}{|c|}{ Arabian } \\
\hline $\mathrm{N}$ & $\%$ & $\mathrm{~N}$ & $\%$ & $\mathrm{~N}$ & $\%$ & $\mathrm{~N}$ & $\%$ & $\mathrm{~N}$ & $\%$ & $\mathrm{~N}$ & $\%$ & $\mathrm{~N}$ & $\%$ & $\mathrm{~N}$ & $\%$ \\
\hline 0 & $0 \%$ & 3 & $4.23 \%$ & 0 & $0 \%$ & 4 & $5.63 \%$ & 0 & $0 \%$ & 4 & $5.56 \%$ & 0 & $0 \%$ & 5 & $6.94 \%$ \\
\hline 2 & $4.35 \%$ & 6 & $8.45 \%$ & 2 & $4.35 \%$ & 2 & $2.82 \%$ & 6 & $13.04 \%$ & 3 & $4.17 \%$ & 8 & $17.39 \%$ & 7 & $9.72 \%$ \\
\hline 5 & $10.87 \%$ & 9 & $12.68 \%$ & 2 & $4.35 \%$ & 9 & $12.68 \%$ & 10 & $21.74 \%$ & 10 & $13.89 \%$ & 7 & $15.22 \%$ & 7 & $9.72 \%$ \\
\hline 7 & $15.22 \%$ & 7 & $9.86 \%$ & 0 & $0 \%$ & 3 & $4,23 \%$ & 2 & $4.35 \%$ & 7 & $9.72 \%$ & 3 & $6.52 \%$ & 11 & $15.28 \%$ \\
\hline 7 & $15.22 \%$ & 6 & $8.45 \%$ & 7 & $15.22 \%$ & 7 & $9.86 \%$ & 6 & $13.04 \%$ & 15 & $20.83 \%$ & 8 & $17.39 \%$ & 14 & $19.44 \%$ \\
\hline 10 & $21.74 \%$ & 13 & $18.31 \%$ & 3 & $6.52 \%$ & 7 & $9.86 \%$ & 4 & $8.70 \%$ & 9 & $12.50 \%$ & 6 & $13.04 \%$ & 6 & $8.33 \%$ \\
\hline 5 & $10.87 \%$ & 12 & $16.90 \%$ & 7 & $15.22 \%$ & 6 & $8.45 \%$ & 12 & $26.09 \%$ & 8 & $11.11 \%$ & 13 & $28.26 \%$ & 10 & $13.89 \%$ \\
\hline 10 & $21.74 \%$ & 10 & $14.08 \%$ & 15 & $32.61 \%$ & 9 & $12.68 \%$ & 3 & $6.52 \%$ & 7 & $9.72 \%$ & 1 & $2.17 \%$ & 3 & $4.17 \%$ \\
\hline 0 & $0 \%$ & 5 & $7.04 \%$ & 9 & $19.57 \%$ & 9 & $12.68 \%$ & 3 & $6.52 \%$ & 3 & $4.17 \%$ & 0 & $0 \%$ & 5 & $6.94 \%$ \\
\hline 0 & $0 \%$ & 0 & $0 \%$ & 1 & $2.17 \%$ & 15 & $21.13 \%$ & 0 & $0 \%$ & 6 & $8.33 \%$ & 0 & $0 \%$ & 4 & $5.56 \%$ \\
\hline
\end{tabular}

Table 3: Frequency and distribution of emotional feeling of mental health providers by patients with mental diseases.

\begin{tabular}{|c|c|c|c|c|c|c|c|c|c|c|c|c|c|c|c|c|}
\hline \multirow{3}{*}{ Feeling } & \multicolumn{4}{|c|}{ Suicide attempts } & \multicolumn{4}{|c|}{ Depressive disorders } & \multicolumn{4}{|c|}{ Psychosis } & \multicolumn{4}{|c|}{ Sexual abuse } \\
\hline & \multicolumn{2}{|c|}{ Americans } & \multicolumn{2}{|c|}{ Arabian } & \multicolumn{2}{|c|}{ Americans } & \multicolumn{2}{|c|}{ Arabian } & \multicolumn{2}{|c|}{ Americans } & \multicolumn{2}{|c|}{ Arabian } & \multicolumn{2}{|c|}{ Americans } & \multicolumn{2}{|c|}{ Arabian } \\
\hline & $\mathrm{N}$ & $\%$ & $\mathrm{~N}$ & $\%$ & $\mathrm{~N}$ & $\%$ & $\mathrm{~N}$ & $\%$ & $\mathrm{~N}$ & $\%$ & $\mathrm{~N}$ & $\%$ & $\mathrm{~N}$ & $\%$ & $\mathrm{~N}$ & $\%$ \\
\hline Anger & 28 & $62.22 \%$ & 22 & $32.64 \%$ & 1 & $2.22 \%$ & 4 & $6.06 \%$ & 5 & $11.11 \%$ & 5 & $7.58 \%$ & 22 & $48.89 \%$ & 34 & $52.31 \%$ \\
\hline $\begin{array}{c}\text { Frustra } \\
\text { tion }\end{array}$ & 36 & $80 \%$ & 28 & $41.79 \%$ & 6 & $13.33 \%$ & 4 & $6.06 \%$ & 7 & $15.56 \%$ & 10 & $15.15 \%$ & 6 & $13.33 \%$ & 12 & $18.46 \%$ \\
\hline Sadness & 34 & $75.56 \%$ & 38 & $56.72 \%$ & 22 & $48.89 \%$ & 32 & $48.48 \%$ & 19 & $42.22 \%$ & 15 & $22.73 \%$ & 25 & $25.56 \%$ & 24 & $36.92 \%$ \\
\hline
\end{tabular}




\begin{tabular}{|c|c|c|c|c|c|c|c|c|c|c|c|c|c|c|c|c|}
\hline Guilty & 16 & $35.56 \%$ & 17 & $25.37 \%$ & 2 & $4.44 \%$ & 7 & $10.61 \%$ & 0 & $0 \%$ & 3 & $4.55 \%$ & 0 & $0 \%$ & 7 & $10.77 \%$ \\
\hline Empathy & 39 & $86.67 \%$ & 36 & $53.73 \%$ & 44 & $97.78 \%$ & 48 & $72.73 \%$ & 40 & $88.89 \%$ & 33 & $50 \%$ & 42 & $93.33 \%$ & 31 & $47.69 \%$ \\
\hline Disgust & 0 & $0 \%$ & 0 & $0 \%$ & 0 & $0 \%$ & 1 & $1.52 \%$ & 0 & $0 \%$ & 1 & $1.52 \%$ & 4 & $8.89 \%$ & 8 & $12.31 \%$ \\
\hline $\begin{array}{c}\text { Fear or } \\
\text { nervou } \\
\text { sness }\end{array}$ & 6 & $13.33 \%$ & 6 & $8.96 \%$ & 0 & $0 \%$ & 7 & $10.61 \%$ & 3 & $6.67 \%$ & 6 & $9.09 \%$ & 13 & $28.89 \%$ & 11 & $16.92 \%$ \\
\hline $\begin{array}{l}\text { No parti } \\
\text { cular } \\
\text { feelings }\end{array}$ & 1 & $2.22 \%$ & 6 & $8.96 \%$ & 1 & 2.22 & 3 & $4.55 \%$ & 1 & $2.22 \%$ & 16 & $24.24 \%$ & 0 & $0 \%$ & 5 & $7.69 \%$ \\
\hline
\end{tabular}

\begin{tabular}{|c|c|c|c|c|c|c|c|c|c|c|c|c|c|c|c|}
\hline \multicolumn{4}{|c|}{ Physical abuse } & \multicolumn{4}{|c|}{ Substance Abuse } & \multicolumn{4}{|c|}{ Bipolar Disorder } & \multicolumn{4}{|c|}{ Anxiety Disorders } \\
\hline \multicolumn{2}{|c|}{ American } & \multicolumn{2}{|c|}{ Arabian } & \multicolumn{2}{|c|}{ American } & \multicolumn{2}{|c|}{ Arabian } & \multicolumn{2}{|c|}{ American } & \multicolumn{2}{|c|}{ Arabian } & \multicolumn{2}{|c|}{ American } & \multicolumn{2}{|c|}{ Arabian } \\
\hline $\mathrm{N}$ & $\%$ & $\mathrm{~N}$ & $\%$ & $\mathrm{~N}$ & $\%$ & $\mathrm{~N}$ & $\%$ & $\mathrm{~N}$ & $\%$ & $\mathrm{~N}$ & $\%$ & $\mathrm{~N}$ & $\%$ & $\mathrm{~N}$ & $\%$ \\
\hline 24 & $53.33 \%$ & 39 & $60 \%$ & 9 & $20 \%$ & 10 & $15.87 \%$ & 2 & $4.35 \%$ & 6 & $9.52 \%$ & 1 & $2.17 \%$ & 4 & $6.15 \%$ \\
\hline 7 & $15.56 \%$ & 9 & $13.85 \%$ & 16 & $35.56 \%$ & 10 & $15.87 \%$ & 6 & 13.04 & 9 & $14.29 \%$ & 5 & $10.87 \%$ & 8 & $12.31 \%$ \\
\hline 24 & $53.33 \%$ & 16 & $24.62 \%$ & 13 & $28.89 \%$ & 7 & $11.11 \%$ & 11 & $23.91 \%$ & 9 & $14.29 \%$ & 10 & $21.74 \%$ & 12 & $18.46 \%$ \\
\hline 1 & $2.22 \%$ & 4 & $6.15 \%$ & 2 & 4 & 3 & $4.76 \%$ & 1 & $2.17 \%$ & 2 & $3.17 \%$ & 0 & $0 \%$ & 1 & $1.54 \%$ \\
\hline 40 & $88.89 \%$ & 32 & $49.23 \%$ & 26 & $57.78 \%$ & 24 & $38.10 \%$ & 39 & $84.78 \%$ & 39 & $61.90 \%$ & 29 & $63.04 \%$ & 36 & $55.38 \%$ \\
\hline 2 & $4.44 \%$ & 4 & $6.15 \%$ & 3 & $6.67 \%$ & 6 & 9.52 & 0 & $0 \%$ & 4 & $6.35 \%$ & 0 & $0 \%$ & 2 & $3.08 \%$ \\
\hline 9 & $20 \%$ & 5 & $7.69 \%$ & 1 & $2.22 \%$ & 0 & $0 \%$ & 3 & $6.52 \%$ & 7 & $11.11 \%$ & 0 & $0 \%$ & 6 & $9.23 \%$ \\
\hline 0 & $0 \%$ & 4 & $6.15 \%$ & 13 & $28.89 \%$ & 19 & $30.16 \%$ & 4 & $8.70 \%$ & 9 & $14.29 \%$ & 17 & $36.96 \%$ & 13 & $20 \%$ \\
\hline
\end{tabular}

\begin{tabular}{|c|c|c|c|c|c|c|c|c|c|c|c|c|c|c|c|}
\hline \multicolumn{4}{|c|}{ ssssssss } & \multicolumn{4}{|c|}{ Childhood Psychiatric Disorders } & \multicolumn{4}{|c|}{ Personality Disorders } & \multicolumn{4}{|c|}{ Deliberate Self-Harm } \\
\hline \multicolumn{2}{|c|}{ American } & \multicolumn{2}{|c|}{ Arabian } & \multicolumn{2}{|c|}{ American } & \multicolumn{2}{|c|}{ Arabian } & \multicolumn{2}{|c|}{ American } & \multicolumn{2}{|c|}{ Arabian } & \multicolumn{2}{|c|}{ American } & \multicolumn{2}{|c|}{ Arabian } \\
\hline $\mathrm{N}$ & $\%$ & $\mathrm{~N}$ & $\%$ & $\mathrm{~N}$ & $\%$ & $\mathrm{~N}$ & $\%$ & $\mathrm{~N}$ & $\%$ & $\mathrm{~N}$ & $\%$ & $\mathrm{~N}$ & $\%$ & $\mathrm{~N}$ & $\%$ \\
\hline 14 & $31.11 \%$ & 14 & $21.54 \%$ & 27 & $61.36 \%$ & 23 & $36.51 \%$ & 11 & $23.91 \%$ & 8 & $12.31 \%$ & 6 & $13.04 \%$ & 9 & $13.85 \%$ \\
\hline 13 & $28.89 \%$ & 18 & $27.69 \%$ & 30 & $68.18 \%$ & 24 & $38.10 \%$ & 18 & $39.13 \%$ & 14 & $21.54 \%$ & 14 & $30.43 \%$ & 12 & $18.46 \%$ \\
\hline 18 & $40 \%$ & 30 & $46.15 \%$ & 25 & $56.82 \%$ & 32 & $50.79 \%$ & 12 & $26.09 \%$ & 9 & $13.85 \%$ & 15 & $32.61 \%$ & 12 & $18.46 \%$ \\
\hline 0 & $0 \%$ & 2 & $3.08 \%$ & 1 & $2.27 \%$ & 4 & $6.35 \%$ & 1 & $2.17 \%$ & 2 & 3.08 & 1 & 2.17 & 3 & $4.62 \%$ \\
\hline 41 & 91.11\% & 43 & $66.15 \%$ & 34 & $77.27 \%$ & 35 & $55.56 \%$ & 23 & $50 \%$ & 23 & $35.38 \%$ & 22 & $47.83 \%$ & 31 & $47.69 \%$ \\
\hline 0 & $0 \%$ & 3 & $4.62 \%$ & 1 & $2.27 \%$ & 3 & $4.76 \%$ & 1 & 2.17 & 12 & $18.46 \%$ & 1 & $2.17 \%$ & 6 & $9.23 \%$ \\
\hline 4 & $8.89 \%$ & 4 & $6.15 \%$ & 6 & $13.64 \%$ & 5 & $7.94 \%$ & 3 & $6.52 \%$ & 5 & $7.69 \%$ & 1 & $2.17 \%$ & 2 & $3.08 \%$ \\
\hline 2 & $4.44 \%$ & 9 & $13.85 \%$ & 3 & $6.82 \%$ & 11 & $17.46 \%$ & 15 & $32.61 \%$ & 19 & $29.23 \%$ & 16 & $34.78 \%$ & 11 & $16.92 \%$ \\
\hline
\end{tabular}

Scoring of the Reactions of Mental Health Providers Towards Psychological Cases of Patients

As shown in Table (2), the reaction of mental health provider as a bothering level was assessed in a scale from 1-10. The peak of bothering level was recorded for the degree $9 / 10$ by $50 \%$ of American participants. On the other hand, 23.29\% of Arabian participants showed the highest level of bothering towards suicide attempts at $100 \%$ level. The bothering level for Americans against depressive disorders was highest (23.91\%) at the degree 6/10, while it was (18.31\%) at the degree 8/10 among Arabians. The reaction of Americans towards psychosis was equally (19.57\%) at three degrees $4 / 10,5 / 10$, and $8 / 10$, whereas the reaction of Arabians was the highest (19.72\%) at the degree 6/10. The reactions of both American and Arabian participants were at peak at the degree 8/10. Regarding physical abuse, among Americans, the highest reaction (23.91\%) was at the middle degree 5/10, while among Arabians, the highest degree (26.76\%) was at the degree
8/10. Among Americans, the reactions against substance abuse was at peak (23.91\%) at two degrees $3 / 10$, and $7 / 10$, whereas among Arabians the highest reaction (20.83\%) was observed at the degree 5/10. For bipolar disorder, and manic episodes, the highest bothering reaction (26.09\%) was recorded at the degree $7 / 10$ among Americans, and the highest reaction of Arabians $(26.39 \%)$ at the degree 5/10. Regarding anxiety disorders, the reactions among Americans were at peak (21.47\%) at the degree $3 / 10$, whereas among Arabians, similar peaks (18.06\%) were observed at three degrees $2 / 10,4 / 10$, and $5 / 10$. For post-traumatic disorders, the highest reactions $(21.74 \%)$ were observed at the degrees $6 / 10$, and $8 / 10$. On the other hand, the highest reaction (18.31\%) was at the degree 6/10 among Arabians. Americans exhibited their bothering feelings towards childhood psychiatric disorders to reach peak (32.61\%) at the degree 8/10. Arabians, on the other hand exhibited the highest levels $(21.13 \%)$ at the degree $10 / 10$. Personality disorders triggered bothering feelings at peak $(26.09 \%)$ at the degree of $7 / 10$ for Americans, and $(20.83 \%)$ at 
the degree of 5/10 for Arabians. The data presented in table (2) showed that the Americans were bothered by deliberate self-harm at peak (28.26\%) at the degree of $7 / 10$, whereas among Arabians, the peak was (19.44\%) at the degree of 5/10.

\section{Types of Feelings of Mental Health Providers Treating Patients with Mental Health Problems}

As shown in Table (3), eight emotional feelings were investigated and compared among Americans and Arabians who are involved in treating patients with mental diseases. The emotional impacts of suicide attempts by Americans varied in intensity in which empathy was the highest (86.7\%), frustration (80\%), while disgust was $(0 \%)$. Among Arabians, the most prevalent feeling was sadness $(56.72 \%)$, and disgust was (0\%). Regarding depressive disorders, empathy was the most prevalent feeling (97.78\%) among Americans, followed by sadness (48.89\%), while among Arabians, empathy was the most prevalent feeling $(72.73 \%)$ followed by sadness (48.48\%). Regarding psychosis, the most prevalent feeling was empathy among Americans (88.89\%), and Arabians (50\%). For sexual abuse, the most prevalent feeling was empathy among Americans (93.33\%), and anger among Arabians (52.31\%). Regarding physical abuse, Americans expressed the feeling of empathy (88.89\%), while Arabians expressed the anger feelings by $(60 \%)$ of participants. The empathy feelings were highest by Americans (57.78\%) and Arabians (38.1\%) for substance abuse. For bipolar disorders and manic episodes, empathy was the most prevalent feeling (84.78\%) exhibited by Americans, and it was also the most prevalent feeling among Arabians (61.90\%). For anxiety disorders, empathy was the mostly prevalent feeling among Americans (63.04\%), and Arabians (55.38\%). For post-traumatic stress disorders, the most prevalent feeling was empathy among both Americans (91.11\%), and Arabians (66.15\%). For childhood psychiatric disorders, empathy was the mostly prevalent among Americans (77.27\%) and Arabians (55.56\%). For personality disorders, empathy was the mostly prevalent among Americans (50\%) and Arabians (35.38\%). For deliberate self-harm, the most prevalent feeling was empathy among Americans (47.83\%), and Arabians (47.69\%).

\section{Emotional Feeling of Mental Health Providers and their Mechanisms of Interactions}

Mental health providers followed different patterns of behaviors when they thought that their emotional reactions might interfere with their treatment of a particular patient. As shown in Table (4), 100\% of Americans reported talking with a senior colleague or supervisor, whereas $61.19 \%$ of Arabians reported the same. Americans (31.11\%) reported their responses as to engage in more formal treatment with another mental health provider, and this pattern of behavior was more than that reported by Arabians (25.37\%). Reviewing the literature on the disorder and therapist responses was exhibited by similar trends by Americans and Arabians (33.33\%, and 32.84\% respectively). Talking with a non-mental health provider friend informally was reported by 8.89\% Americans and $2.99 \%$ Arabians. Talking with your spouse was reported by $62.22 \%$ Americans, and $25.37 \%$ Arabians. Finally,
$2.22 \%$ of Americans, and $13.43 \%$ of Arabians reported that no particular response is necessary once one is aware of these feelings.

\section{Discussion}

The present study was conducted to explore the extent to which emotional feelings of mental health providers towards patients with mental diseases have developed among health professionals from origins of Arabians and Americans. The data showed that both Americans and Arabians exhibited degrees of bothering towards patients with mental diseases. In this context, it was found that in general terms there are bothering levels at varying degrees among Americans and Arabians towards such patients which can be understood as accumulative impacts resulting from the experience with such patients. However, this trend has been described in the literature through various health providing settings and by various mental health providing groups including nurses and psychiatrists $[2,9]$.

A focus has been made on eight emotions to which extent they were distributed towards patients with mental diseases. Taken into consideration the suicide attempts, the Americans exhibited maximally empathy, whereas Arabians exhibited sadness. Either sadness or empathy are not considered negative emotional feelings, but rather from humanitarian aspects, such patients developed understanding on mental health providers. Empathy was reported in several studies [7-9]. Regarding depressive disorders, psychosis, bipolar disorders and manic episodes, anxiety disorders, posttraumatic stress disorders, childhood psychiatric disorders, personality disorders, deliberate self-harm and substance abuse, empathy was the most prevalent feeling among both Americans and Arabians, which can be explained as in the previous mental diseases. Sexual abuse and physical abuse triggered empathy among Americans and Anger among Arabians as the most prevalent emotional feelings. We think culture and religion play a role in this context. These findings indicated that although the Americans and Arabians were bothered by actions of mental patients, they understood their patients. The mechanisms of interaction with the patients in case of developing negative feelings varied among Americans and Arabians. It seems the controlling systems and underlying work environment play a great role.

\section{Conclusion}

The present study showed that both Americans and Arabians developed bothering levels of actions of mental patients and exhibited different levels of empathy towards those patients.

\section{References}

1. Lauber C, Nordt C, Braunscheig C, Rossler W (2006) Do mental health professionals stigmatize their patients? Acta Psychiatrica Scandanavia 113(429): 51-59.

2. Deans C, Meocivic E (2006) Attitudes of registered psychiatric nurses towards patients diagnosed with borderline personality disorder. Contemporary Nurse 21(1): 43-49.

3. James PD, Cowman S (2007) Psychiatric nurses' knowledge, experience, and attitudes towards clients with borderline personality disorder. Journal of Psychiatric and Mental Health Nursing 14(7): 670-678. 
4. Servais LM, Saunders SM (2007) Clinical psychologists' perceptions of persons with mental illness. Professional Psychology: Research and Practice 38(2): 214-219.

5. Ucok A, Polat A, Sartorius N, Erkoc S, Atakli C (2004) Attitudes of psychiatrists toward patients with schizophrenia. Psychiatry and Clinical Neurosciences 58(1): 89-91.

6. Bjorkman T, Angelman T, Jonsson M (2008) Attitudes towards people with mental illness: A cross-sectional study among nursing staff in psychiatric and somatic care. Scandinavian Journal of Caring Science 22(2): 170-177.

7. Decety J (2009) Empathy, sympathy and the perception of pain. Pain 145(3): 365-366

ISSN: 2574-1241

DOI: $10.26717 / B J S T R .2018 .09 .001776$

Raafat Aburumman. Biomed J Sci \& Tech Res

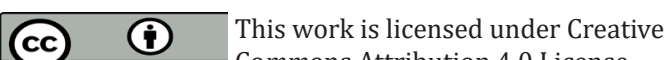

Submission Link: https://biomedres.us/submit-manuscript.php
8. Decety J, Jackson PL (2004) The functional architecture of human empathy. Behavioral and cognitive neuroscience reviews 3(2): 71-100.

9. Hernando Santamaría-García, Sandra Baez, Adolfo M García, Daniel Flichtentrei, María Prats, et al. (2017) Empathy for others' suffering and its mediators in mental health professionals. Scientific reports 7: 6391.

10. Carlozzi AF, Bull KS, Stein LB, Ray K, Barnes L (2002) Empathy theory and practice: A survey of psychologists and counselors. The Journal of psychology 136(2): 161-170.

11. Singer T, Lamm C (2009) The social neuroscience of empathy. Annals of the New York Academy of Sciences 1156: 81-96.

12. de Waal FBM (2012) The antiquity of empathy. Science 336(6083): 874876.

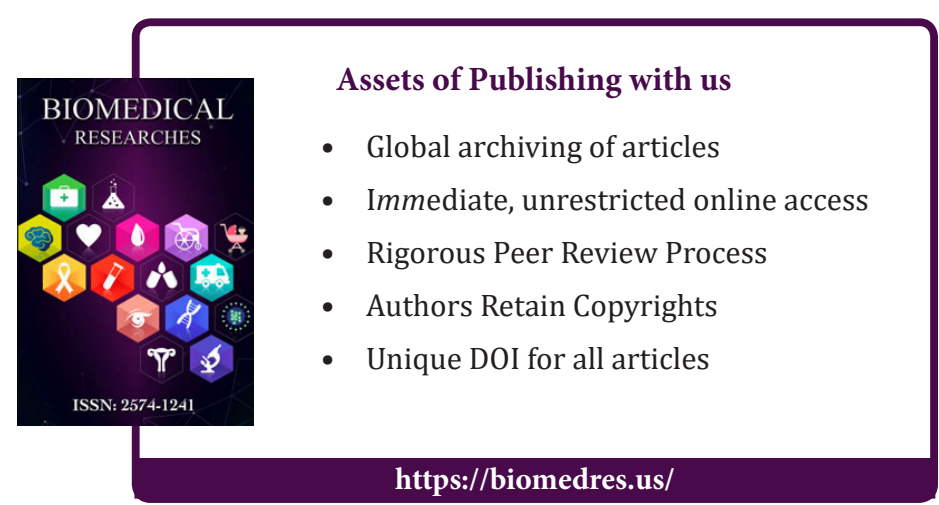

